\title{
Embedding non-rigid solutes in an averaged environment: a case study on rhodopsins
}

\author{
Niccolò Ricardi, ${ }^{* \dagger}$ Cristina E. González-Espinoza, ${ }^{\dagger}$ Suliman Adam ${ }^{\ddagger}{ }^{\ddagger}$ Jonathan

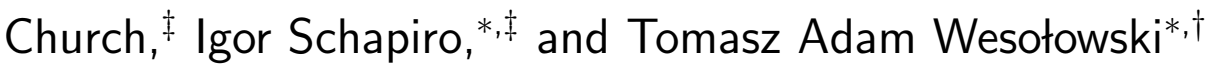 \\ $\dagger$ Department of Physical Chemistry, University of Geneva, Geneva (Switzerland) \\ $\ddagger$ Fritz Haber Center for Molecular Dynamics, Hebrew University of Jerusalem Israel, \\ Jerusalem (Israel)
}

E-mail: Niccolo.Ricardi@unige.ch; Igor.Schapiro@mail.huji.ac.il; Tomasz.Wesolowski@unige.ch

\section{Abstract}

Many simulation methods concerning solvated molecules are based on the assumption that the solvated species and the solvent can be characterized by some representative structure of the solute and some embedding potential corresponding to this structure. This assumption is re-examined and generalized for conformationally flexible solutes. In the proposed and investigated generalization, the solute is characterized by a set of representative structures and the corresponding embedding potentials. The representative structures are identified by means of subdividing the statistical ensemble, which in this work is generated by a constant-temperature molecular dynamics simulation. The embedding potential defined in Frozen-Density Embedding Theory is used to characterize the average effect of the solvent in each subensemble. The numerical examples concern vertical excitation energies of protonated retinal Schiff bases in protein environments. It is comprehensively shown that subensemble averaging leads to huge computational savings compared to explicit averaging of the excitation energies in the whole ensemble while introducing only minor errors.

\section{Introduction}

Modeling molecules in the condensed phase requires simulation of large systems. This has led to the development of a multitude of multiscale approaches. These differ greatly, but most of them are based on the division of the system into two subsystems: the system of interest, from hereon denoted as A, and the environment, from hereon denoted as B. Another challenging aspect is the need to extend the calculation also on the timescale, to account for the thermal agitation of the system. Sampling of the statistical ensemble, by means of molecular dynamics or molecular Monte Carlo, can be used to obtain a set of geometries $\left\{\mathbf{R}^{i}\right\}$ (divided into $\left\{\mathbf{R}_{A}^{i}\right\}$ and $\left\{\mathbf{R}_{B}^{i}\right\}$ ) which adequately sample the free energy surface. The ensemble of observables $\left\{\left\langle\hat{O}_{\mathbf{R}_{A}^{i}, \mathbf{R}_{B}^{i}}\right\rangle\right\}$ - where the subscript denotes the coordinates used to calculate the observable - can then be investigated. For the sake of simplicity, a single average value $\left\langle\left\langle\hat{O}_{\mathbf{R}_{A}^{i}, \mathbf{R}_{B}^{i}}\right\rangle\right\rangle_{N}$ may be considered, where $\mathrm{N}$ is the number of samples of the statistical ensemble. Directly computing such a value would constitute a significant computational advantage. To this end, a single solute structure representation is commonly used, resulting in:

$$
\left\langle\left\langle\hat{O}_{\mathbf{R}_{A}, \mathbf{R}_{B}^{i}}\right\rangle\right\rangle_{N} \approx\left\langle\hat{O}_{\mathbf{R}_{A}, \bar{B}}\right\rangle,
$$


where $\bar{B}$ generically denotes some descriptor of the average environment. This can be done in several ways, which will now be outlined.

For instance in continuum dielectric based solvent models $\bar{B}$ is represented by means of the reaction field. ${ }^{1-3}$

At the atomistic representation of the solvent - i.e. if the ensemble of instantaneous structures $\left\{\mathbf{R}_{B}^{i}\right\}$ is available - $\bar{B}$ is the average electric potential. Such as the ones used in Refs. 4-17.

Frozen-Density Embedding Theory (FDET) ${ }^{18,19}$ provides a straightforward interpretation of $\bar{B}$ that includes not only electrostatics but a complete quantum-mechanical description of the whole system. In FDET, $\bar{B}$ represents two quantities: the statistically averaged electrostatic potential generated by the nuclei of the solvent $\left\langle v_{B}\right\rangle(\mathbf{r})$ and the statistically averaged electron density of the environment $\left\langle\rho_{B}\right\rangle(\mathbf{r})$. The FDET embedding potential in such a case reads: ${ }^{20}$

$$
\begin{aligned}
& v_{\mathrm{emb}}\left[\rho_{A},\left\langle\rho_{B}\right\rangle ;\left\langle v_{B}\right\rangle\right](\mathbf{r})=\left\langle v_{B}\right\rangle(\mathbf{r})+ \\
& \int \frac{\left\langle\rho_{B}\right\rangle\left(\mathbf{r}^{\prime}\right)}{\left|\mathbf{r}-\mathbf{r}^{\prime}\right|} d \mathbf{r}^{\prime}+v_{x c T}^{\text {nadd }}\left[\rho_{\mathrm{A}},\left\langle\rho_{B}\right\rangle\right](\mathbf{r}),
\end{aligned}
$$

where the first two terms account for the electrostatics (nuclear attraction and Coulomb repulsion) and the last models with orbital-free density functional theory the exchange, kinetic, and correlation interaction between the two subsystems.

There may be several reasons to justify the use of a single solute structure representation approach to model subsystem A ( $\mathbf{R}_{A}$ in Eq. 1). Semi-rigid molecules can simply be modeled by means of their gas-phase structures. ${ }^{20-24} \mathrm{In}$ other cases a structure is chosen which minimizes the free energy of the supersystem, with some multiscale method of choice. ${ }^{5-9,11-17}$

Albeit technically applicable to a set of local free energy minima, ${ }^{25,26}$ the latter approach is commonly applied to the global minimum only, relying on the assumption that the whole ensemble is well represented by a single structure within its average environment. Even when a set of structures is used, their observables are often individually compared to exper- imental values, ${ }^{27}$ rather than obtaining a single Boltzmann-weighted average value.

A more general form of Eq. 1 for non-rigid solutes, where the also the solute coordinates are averaged, reads:

$$
\left\langle\left\langle\hat{O}_{\mathbf{R}_{A}^{i}, \mathbf{R}_{B}^{i}}\right\rangle\right\rangle_{N} \stackrel{?}{=}\left\langle\hat{O}_{\left\langle\mathbf{R}_{A}\right\rangle, \bar{B}}\right\rangle .
$$

It can be reasonably expected the averaging of coordinates, performed in a yet-to-define way, to be sensible when only small fluctuations around a minimum-energy structure are observed, but issues may arise when the solute is flexible. A single average solute structure may be chemically unreasonable or significantly different from any of the conformers, with obvious risks for the quality of any observable pertaining to the structure. Direct averaging of a coordinate spanning over two conformers leads to a value similar to that expected for the transition state for the conformation change. For instance, let us imagine a system with a torsional angle $\Phi$ with two stable conformers characterized by the values $\Phi_{1}$ and $\Phi_{2}$ with very similar free energies $G_{1} \approx G_{2}$. The occurrence of the two conformers will be approximately equal, resulting in an average value $\Phi_{\text {avg }} \approx \frac{\Phi_{1}+\Phi_{2}}{2}$, which is in turn similar to the angle, and consequently energy, of the transition state for the conformerism. Furthermore, the movement of the environment will generally be coupled to the movement of the solute, and failure to account for this may prevent the average environment from having a proper cavity for the solute. This can be due to steric effects, such as the cavity adapting to the flexible solute. Also, if the instantaneous structures oscillate around average one which has a higher symmetry, this approximation is expected to fail. For instance, quadrupolar solutes can assume an instantaneous dipole moment, which in turn can induce order in the environment.

One possible strategy to avoid such issues is to divide the statiscal ensemble into subensembles according to

$$
\left\langle\left\langle\hat{O}_{\mathbf{R}_{A}^{i}, \mathbf{R}_{B}^{i}}\right\rangle\right\rangle_{N}=\sum_{k=1}^{K}\left\langle\hat{O}_{\mathbf{R}_{A}^{i}, \mathbf{R}_{B}^{i}}\right\rangle_{N_{k}} \cdot \frac{N_{k}}{N},
$$


where $N_{k}$ is the number of samples in subensemble $k$, and then apply the approximation in Eq. 3 to each subensemble, where it can be expected to be reasonably accurate, obtaining

$$
\left\langle\left\langle\hat{O}_{\mathbf{R}_{A}^{i}, \mathbf{R}_{B}^{i}}\right\rangle\right\rangle_{N} \approx \sum_{k=1}^{K}\left\langle\hat{O}_{\left\langle\mathbf{R}_{A}\right\rangle, \bar{B}_{k}}\right\rangle_{k} \cdot \frac{N_{k}}{N},
$$

which for the specific case of FDET becomes:

$$
\begin{array}{r}
\left.\left\langle\left\langle\hat{O}_{\mathbf{R}_{A}^{i}, \mathbf{R}_{B}^{i}}\right\rangle\left[\rho_{B_{i}}\right]\right\rangle\right\rangle_{N} \approx \\
\sum_{k=1}^{K}\left\langle\hat{O}_{\left\langle\mathbf{R}_{A}\right\rangle_{k},\left\langle v_{B}^{n u c}\right\rangle_{k}}\right\rangle\left[\left\langle\rho_{B}\right\rangle_{k}\right] \cdot \frac{N_{k}}{N} .
\end{array}
$$

If the sampling represents the statistical equilibrium, the relative population in a subensemble is given by:

$$
\frac{N_{k}}{N}=\frac{e^{\frac{-G_{k}}{k_{B} T}}}{Q}
$$

where $G_{k}$ is the free energy of conformer $k$ and $Q$ is the system's partition function. Introducing this in Eq. 5 one gets:

$$
\left\langle\left\langle\hat{O}_{\mathbf{R}_{A}^{i}, \mathbf{R}_{B}^{i}}\right\rangle\right\rangle_{N} \approx \sum_{k=1}^{K}\left\langle\hat{O}_{\left\langle\mathbf{R}_{A}\right\rangle, \bar{B}_{k}}\right\rangle_{k} \cdot \frac{e^{\frac{-G_{k}}{k_{B} T}}}{Q} .
$$

Eq. 4 is exact regardless of how the division into $K$ subensembles is performed. The approximation in Eq. 5 may depend strongly on the specifics of the division into subensembles. The focus of this manuscript is to assess the accuracy of the approximation in Eq. 5 for the specific case of subensembles identified by the conformational changes of the system.

Various methods of clustering conformations were developed in different fields and can yield subensembles. ${ }^{28-46}$ Most clustering algorithms use a top-down approach where the overall similarity of structures, or a subset thereof, is used as the main criterion. Albeit the use of clustering algorithms to average geometries may be a valid and promising strategy, we used a bottomup approach based on the monitoring of the individual behavior of each internal coordinate: the trajectories of individual internal coordinates were analyzed, clustered, compared, and finally grouped to obtain the major conformation changes. This allowed monitoring of the individual behavior of each internal coordinate.

The averaging of both solute and environment is performed within each subensemble defined by the conformation change, from hereon called "conformational basin", according to Eq. 3. If $K$ conformational basins are present, the final observable value is obtained as the weighted average of the values from the $K$ average solute structures, weighted by the population $N_{k}$ of each conformational basin (cf. Eq 5).

For the sake of brevity, a simplified notation will be used from hereon: the quantum mechanical averaging to obtain the expectation value will be omitted, and observables obtained from a single calculation will be denoted as

$$
O_{i}=\left\langle\hat{O}_{\mathbf{R}_{A}^{i}, \mathbf{R}_{B}^{i}}\right\rangle
$$

for individual samples, and

$$
\tilde{O}_{k}=\left\langle\hat{O}_{\left\langle\mathbf{R}_{A}\right\rangle_{k}, \bar{B}_{k}}\right\rangle
$$

when the average solute structure within its average environment, both for conformational basin $k$, is used. The symbols " \langle\rangle " will then represent the averaging of values obtained from separate quantum mechanical calculations, and their subscript will denote the ensemble or subensemble over which the averaging takes place.

For our numerical testing we will use the specific case of the distribution of the vertical excitation energy $\varepsilon$ and the correponding oscillator strength $f$. Such distribution allows to recover the inhomogeneous broadening of the spectrum, albeit not homogeneous broadening effects such as vibronic coupling and quantum decoherence. In such case the expectation val- 
ues for the whole ensemble are obtained as:

$$
\begin{aligned}
\langle\varepsilon\rangle_{N}= & \frac{\sum_{i=1}^{N} f_{i} \cdot \varepsilon_{i}}{\sum_{i=1}^{N} f_{i}} \\
\langle f\rangle_{N} & =\frac{\sum_{i=1}^{N} f_{i}}{N} .
\end{aligned}
$$

This procedure is referred to as "ensemble averaging". Conversely, the use of few solute structures embedded in their average environment will be termed "basin-based geometric averaging". Within a single conformational basin, where small oscillations around an average solute structure are assumed, the approximation of Eq. 3 is applied and excitation energies and oscillator strengths are simply calculated for the average solute structure for that basin in its average environment:

$$
\begin{aligned}
\langle\varepsilon\rangle_{k} & \approx \tilde{\varepsilon}_{k} \\
\langle f\rangle_{k} & \approx \tilde{f}_{k} .
\end{aligned}
$$

The averaging of the $\mathrm{K}$ basins $\{k\}$ with populations $N_{k}$ is then performed, and Eq. 5 for our chosen observables becomes:

$$
\begin{aligned}
\langle\varepsilon\rangle_{N} \approx\left\langle\tilde{\varepsilon}_{k}\right\rangle_{1, \ldots, K}=\frac{\sum_{k=1}^{K} N_{k} \cdot \tilde{f}_{k} \cdot \tilde{\varepsilon}_{k}}{\sum_{k=1}^{K} N_{k} \cdot \tilde{f}_{k}} \\
\langle f\rangle_{N} \approx\left\langle\tilde{f}_{k}\right\rangle_{1, \ldots, K}=\quad \frac{\sum_{k=1}^{K} N_{k} \cdot \tilde{f}_{k}}{N} .
\end{aligned}
$$

The principal area of applicability of basinbased geometric averaging is to obtain a single average value from subensembles based on clustering of geometric descriptors and which correspond to metastable structures. For instance torsional angles, hydrogen bonding patterns, coordination number, etc... The proposed procedure may nonetheless be used in a more general context to validate the relation between a geometric descriptor and a quantummechanical observable. An example of such generalized application is the analysis of the correlation between bond length alternation (BLA) and excitation energy, discussed in Section 3.3.

\section{Computational details}

For this analysis, 4 systems consisting of a protonated retinal Schiff base in a protein environment were considered. The systems differed both in the retinal configuration and in the protein environment.

The first test case consists in the all-trans retinal chromophore is embedded in the channelrhodopsin chimera $\mathrm{C} 1 \mathrm{C} 2,{ }^{47}$ and we use $1 \mathrm{~ns}$ of a previously published quantum mechanics / molecular mechanics (QM/MM) molecular dynamics (MD) trajectory (monomer 1 with deprotonated residue E162). ${ }^{48}$ This system is denoted as $A T-C 1 C 2$. We then turned our attention to the mutated jumping spider rhodpsin-1 (JSR1). ${ }^{49-52}$ We analyzed 1 ns QM/MM MD trajectories performed for the all-trans, 9-cis, and 11-cis configurations. These are respectively denoted by $A T$-JSR1, 9C-JSR1, $11 C$ JSR1.

For all calculations, only the first excitation was considered and frames were extracted in 1 ps steps. For $A T-C 1 C 2$, the last 750 ps of the trajectory were used for our analysis, while for JSR1, the full trajectories were used. For each frame, the protein backbone was removed and the residues were capped with hydrogen atoms. Each frame was then aligned to the first one. This is done via a translation to the geometric center and rotation to minimize root mean standard deviation (Kabsch algorithm $\left.{ }^{53,54}\right)$. Superpositions of molecules for visualization were performed in the same fashion. Root-mean-square deviations (RMSD) throughout this manuscript are calculated with all atoms, except where specifically mentioned. In order to perform ensemble averaging, which will be our reference, two excited-state calculations per frame were performed: one in the presence of an embedding potential $(\mathrm{emb})$ and one without (iso).

The embedding potential, which is obtained 
from the linearized-FDET approximation, ${ }^{55,56}$ was obtained with Q-Chem 5.4's ${ }^{57}$ implementation of Frozen Density Embedding Theory, ${ }^{58}$ using the Thomas-Fermi ${ }^{59,60}$ kinetic functional for $v_{T}^{\text {nadd }}\left[\rho_{A}, \rho_{B}\right]$, the Dirac-Slater ${ }^{61}$ functional for $v_{x}^{\text {nadd }}\left[\rho_{A}, \rho_{B}\right]$, and the Vosko-Wilk-Nusair ${ }^{62}$ parametrization of the LSDA correlation energy for $v_{c}^{\text {nadd }}\left[\rho_{A}, \rho_{B}\right]$. The Hartree-Fock density of the isolated protonated Schiff base was used as $\rho_{A}^{r e f}$. The environment density $\rho_{B}$ was obtained as the sum of the isolated Hartree-Fock densities of each residue having at least an atom within $5 \AA$ of the retinal Schiff base, while residues between 5 and 12 $\AA$ away from the chromophore were modeled by means of their force field atomic charges, i.e. CHARMM36 ${ }^{63,64}$ for AT-C1C2 and Amber ff14SB ${ }^{65}$ for JSR1. Both the embedded and isolated excited state calculations were performed with the algebraic diagrammatic construction for the prepolarization propagator first order $(\mathrm{ADC}(1))$, which yields the same excitation energies as configuration interaction singles, but improved oscillator strengths. ${ }^{66}$ The cc-pVDZ ${ }^{67}$ basis set was used. The resolution of identity with the RIMP2-cc-pVDZ as auxiliary basis set was used. ${ }^{68,69}$ We decided to use a qualitatively, albeit not quantitatively, accurate method in order to investigate the accuracy of Eq. 15 and Eq. 16 in order to reduce the computational effort, considering that the focus of this article is the quality of approximation in Eq. 15 and Eq. 16, rather than the comparison to experimental values.

A detailed explanation or review of clustering algorithms lies outside the scope of this work. Most methods treat each structure as a point in a $n$-dimensional space, where $n$ is the number of parameters considered, generally a set of coordinates, and then recognize clusters of point based on some distance metric. The results from trajectory clustering depend strongly on the clustering algorithm, on the distance metric used, and particularly on the selected atoms that it is applied to. ${ }^{31}$ This is why for this numerical test cases we instead developed tools to aid the analysis of the individual trajectories of each internal coordinate.

The detection of these conformational changes was restricted to the solute only, but this may also be extended to the interaction of the solute with the solvent. For instance, different coordination numbers, distance, or partners, may be detected throughout the trajectory.

The trajectory $\left\{\mathbf{R}_{A}^{i}\right\}$, aligned as previously mentioned, was converted to internal coordinates, which we deemed more insightful than cartesian ones. This was performed with the Python module chemcoord. ${ }^{70}$ The standard deviation of each dihedral angle is then evaluated in the $-180^{\circ}-180^{\circ}$ and $0^{\circ}-360^{\circ}$ ranges. The angles for which the latter is smaller than the former are always analyzed in this range, finally the average values are converted back to the conventional $-180^{\circ}-180^{\circ}$ range. An initial screening of the internal coordinates can be done by analysis of the standard deviation: large values correspond to freely rotating groups, small values correspond to stable chemical groups, while medium values need further analyses. Jumps in the value of an internal coordinate can be detected calculating its average value every $x$ frames, where $x$ is a user chosen integer, and and calculating the change between a set of $x$ values and the previous one. Large absolute values of this change correspond to jumps of value.

Clusters for each internal coordinate can be finally detected in two possible ways:

- via clustering of the distribution, which was performed via $\mathrm{x}$-means, ${ }^{71}$ in its $p y$ clustering ${ }^{72}$ implementation

- by first obtaining time domains via detection of $n_{t}$ frames where the running average changes by more than a threshold $\tau$, then grouping time domains whose average value is significantly close (userdefined threshold)

A combination of both strategies has been used in this work.

Clusters of values for individual coordinates which show clear correlation are grouped into conformational basins (e.g. the clusters of all the dihedrals of the $\beta$-ionone ring are grouped into two conformational basins). The averaging 
of the internal coordinates is carried out in each conformational basin. Clusters of freely rotating groups (in our case only methyls) do not show any correlation, and were used to obtain their average values within each conformational basin.

Finally, in the averaging of dihedral angles of freely rotating groups, the relative position of atoms is based on their average relative position during the trajectory: enforcing a chemically sound structure. For instance for a methyl group, one hydrogen is chosen, and its average angle assigned as the center of its major cluster, then the two other hydrogens are placed to be $\sim 120^{\circ}$ apart, where the exact value is given from the average over the trajectory. This suite of analytical tools were included in the fdeta $^{73}$ (FDET-Average) Python module.

The first excitation was then calculated at the same level of theory as the individual frames for each average chromophore structure both with and without average embedding potential.

In order to obtain the average embedding potential the atoms in the environment were grouped by type $(t)$ and modeled by means of their effective charge $q_{i}(t)$. Their effective number of electrons is obtained as:

$$
q_{i(t)}^{e}=-q_{i}+Z_{i(t)}
$$

where $Z_{i(t)}$ is the nuclear charge of the atom $i$, of type $t$. For the sake of brevity, from now on only $t$ will be used. The average solvent density at the position of the grid point with index $\alpha$ $\left(\mathbf{r}_{\alpha}=\left\{x_{\alpha}, y_{\alpha}, z_{\alpha}\right\}\right)$ is then evaluated as:

$$
\left\langle\rho_{B}\right\rangle(\alpha)=\sum_{t} q_{t}^{e} g_{t}(\alpha)
$$

where $g_{t}(\alpha)$ is the time-averaged probability of finding a nucleus of type $t$ in the volume element corresponding to the gridpoint $\alpha$, divided by the volume $\nu_{\alpha}$,

$$
g_{t}(\alpha)=\frac{n_{\alpha, t}}{N \nu_{\alpha}} ; \quad \sum_{t, \alpha} g_{t}(\alpha) \nu_{\alpha}=N_{\text {nuc }}
$$

The average electrostatic potential is hence:

$$
\left\langle v_{\text {elst }}\right\rangle(\mathbf{r})=\sum_{t, \alpha} \frac{\left(-q_{t}^{e}+Z_{t}\right) g_{t}(\alpha) \nu_{\alpha}}{\left|\mathbf{r}-\mathbf{r}_{\alpha}\right|}
$$

And finally the embedding potential within linearized-FDET becomes:

$$
\begin{array}{r}
v_{\mathrm{emb}}\left[\rho_{A}^{r e f},\left\langle\rho_{B}\right\rangle ;\left\langle v_{\text {elst }}\right\rangle\right](\mathbf{r})=\left\langle v_{\text {elst }}\right\rangle(\mathbf{r}) \\
+v_{x c, T}\left[\rho_{A}^{r e f},\left\langle\rho_{B}\right\rangle\right](\mathbf{r}) .
\end{array}
$$

Only the frames pertaining to the $k$-th conformational basin used to obtain the solute structure were used to obtain the average embedding potential, guaranteeing to account for any coupling between the motion of the chromophore and of the environment.

The specific bonds used to calculate the bond length alternation (BLA) are shown in the Supporting Information. The range of the BLA values was then divided in 6 equal bins, and the frames grouped based on which bin their BLA pertained to.

In order to estimate how well a specific geometry represents an ensemble or a subensemble, sets of root mean square deviation $(\Theta)$ values were used. These were calculated as:

$$
\Theta_{m, k}=\min _{r o t} \sqrt{\frac{\sum_{l=1}^{n_{\text {atoms }}}\left(\mathbf{x}_{m, l}-\mathbf{x}_{k, l}\right)^{2}}{n_{\text {atoms }}}},
$$

where $\mathbf{x}_{m, l}$ represents the cartesian coordinates $(x, y, z)$ of the $l$-th atom in the geometry of frame $m$ where $m$ spans over the frames of the conformational basin $k$, or all basins for direct geometric averaging, while $\mathbf{x}_{k, l}$ is the $l$-th atom in the average geometry over the conformational basin $k$. The minimization over all possible rotations, after superposition of the geometric centers, is carried out with the Kabsch algorithm. If the index $l$ spans over all atoms, the main contribution will be given by the methyls' rotation, masking any other contribution. Due to this, two different sets of RMSD have been produced: one where $l$ spans over all atoms but methyl hydrogens, and one where it spans only over the atoms of the $\beta$-ionone ring, excluding the methyl hydrogens. 


\section{Results and discussion}

The discussion of our numerical results will be organized as follows: subsection 3.1 will analyze and discuss the detection of the conformational basins and the related average structures, while subsection 3.2 will tackle the comparison of excitation energies and oscillator strengths from average structures to the ensemble of individual values.

Subsection 3.3 concerns the assessment of the relation between a geometrical parameter and an observable by means of basin-based geometric averaging.

\subsection{Structures from geometric averaging}

The major changes in conformation throughout the molecular dynamics simulation were detected by analysis of the trajectories of internal coordinates (cf. Section 2).

Two types of conformerism were detected, to different extent, in the different systems:

- the $\beta$-ionone ring is present in two possible conformations, as foreseable (cf. Fig. 1)

- the conformation of the lysine chain can fluctuate (cf. Fig. 2)

Due to the combined effect of these two motions, two to three conformational basins were analyzed in each system. In the cases of $A T$ JSR 1 and $11 C$-JSR 1 the less frequent conformation of the lysine chain amounted to only 15 and 21 frames respectively. These conformational basins were hence ignored both because such a limited amount of frames may be an insufficient sample for proper averaging and because their weights in Eq. 15 is going to be minimal.

The detected conformational basins are identified as 1,2 , and 3 (when analyzed) with decreasing number of frames pertaining to each conformational basin. Their related average structures are denoted by $\left\langle\mathbf{R}_{A}\right\rangle_{1},\left\langle\mathbf{R}_{A}\right\rangle_{2}$, and $\left\langle\mathbf{R}_{A}\right\rangle_{3}$. As a term of comparison, geometries produced ignoring the division into conformational basins were also produced. This method will be named "direct geometric averaging" and its geometries will be denoted as $\left\langle\mathbf{R}_{A}\right\rangle_{1 \cup \ldots \cup K}$.

A visual comparison of $\left\langle\mathbf{R}_{A}\right\rangle_{1},\left\langle\mathbf{R}_{A}\right\rangle_{2}$, and $\left\langle\mathbf{R}_{A}\right\rangle_{3}$ is given form AT-C1C2 in Fig. 1 and Fig. 2, while similar figures are available for the other systems in the Supporting Information.

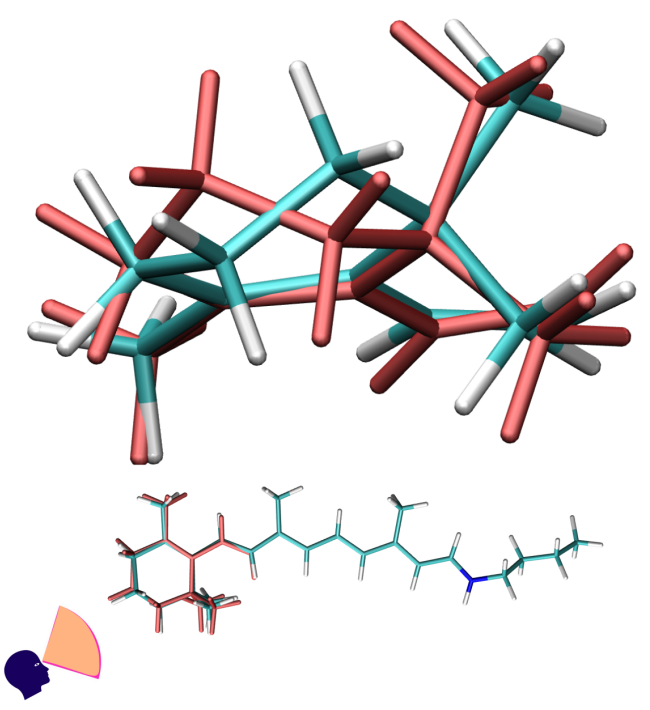

Figure 1: Comparison of $\left\langle\mathbf{R}_{A}\right\rangle_{1}$ and $\left\langle\mathbf{R}_{A}\right\rangle_{2}$ (light red) for AT-C1C2. The geometries' centers have been superimposed and alignment has been performed with the Kabsch algorithm 


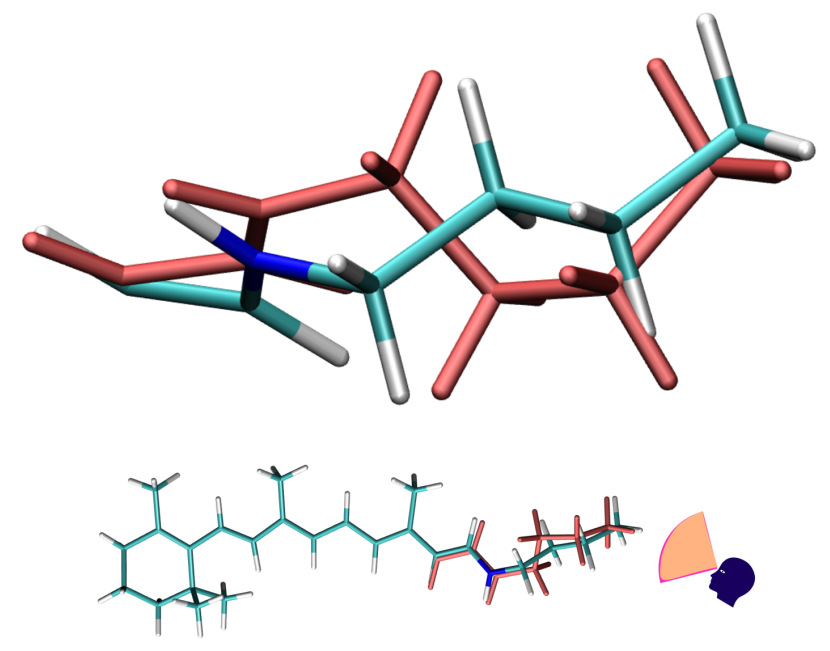

Figure 2: Comparison of superimposed $\left\langle\mathbf{R}_{A}\right\rangle_{1}$ and $\left\langle\mathbf{R}_{A}\right\rangle_{3}$ (light red) for $A T-C 1 C 2$.

Generally, geometries from direct geometric averaging tend to resemble more $\left\langle\mathbf{R}_{A}\right\rangle_{1}$ due to the larger number of frames associated to conformer 1, albeit they still tend to introduce some strain in the $\beta$-ionone ring, due to the mixing of the two conformations. Furthermore, in a cycle, there is always a bond which is not defined in terms of internal coordinates, and which is determined by the position of the atoms: such bond is made unnaturally short by the combination of angles and dihedrals of $\left\langle\mathbf{R}_{A}\right\rangle_{1 \cup 2 \cup 3,[1]}$, which has been obtained with the automatically defined internal coordinates. Such an issue could be detected a posteriori. Nonetheless, it is not possible to have an unphysical combination of dihedrals and angles for a long chain - or a cycle, which is actually described as such in internal coordinates - without it negatively affecting the position of the terminal atom.
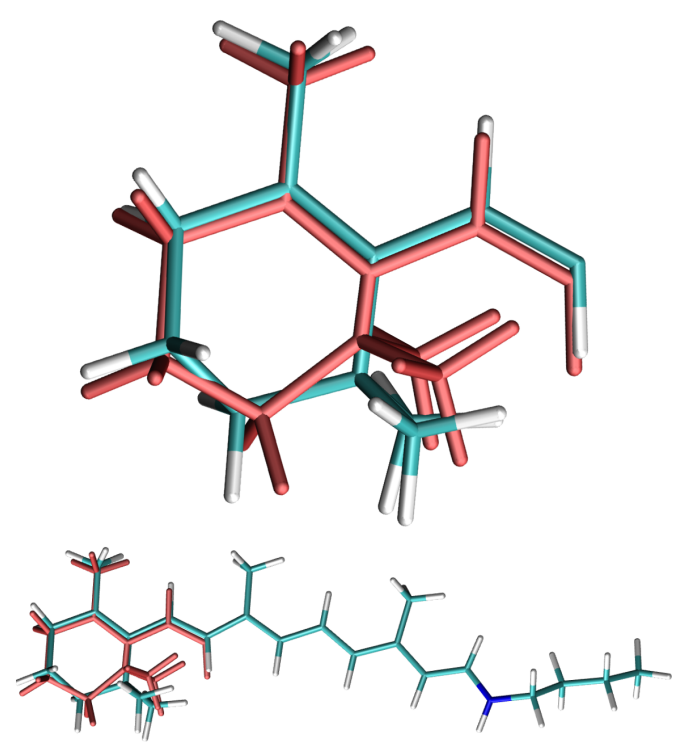

Figure 3: Comparison of superimposed $\left\langle\mathbf{R}_{A}\right\rangle_{1}$ and $\left\langle\mathbf{R}_{A}\right\rangle_{1 \cup 2 \cup 3,[1]}$ (light red) for $A T-C 1 C 2$.
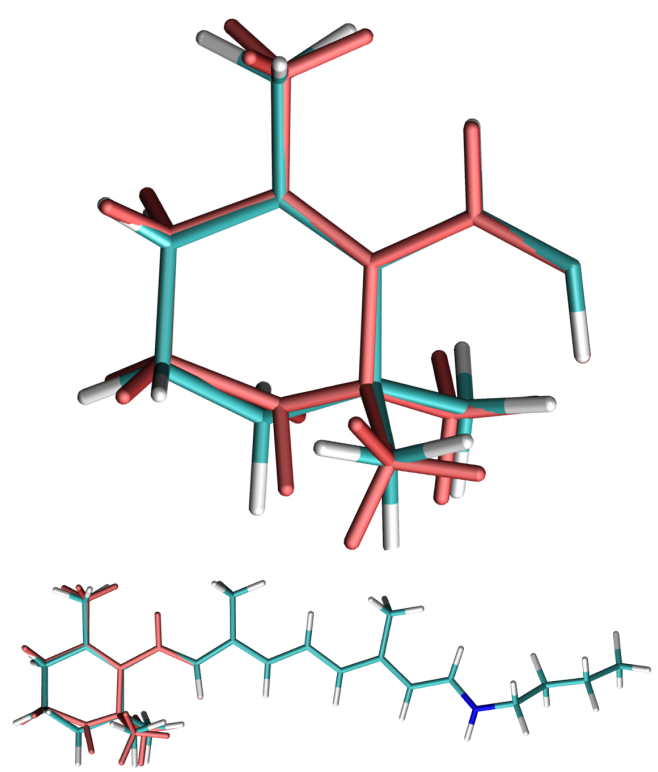

Figure 4: Comparison of superimposed $\left\langle\mathbf{R}_{A}\right\rangle_{1}$ and $\left\langle\mathbf{R}_{A}\right\rangle_{1 \cup 2 \cup 3,[2]}$ (light red) for $A T-C 1 C 2$.

It is on the other hand possible to describe the cycle as two shorter chains rather than a longer one, positioning the undefined bond in the middle. In our case, this also brings the undescribed bond further from the conjugation chain, which is involved in the excitation. In light of this the internal coordinates of the chromophores were defined in such a way that the undefined bond is far from the conjuga- 
tion chain. The geometry from direct geometric averaging with this new set of coordinates is named $\left\langle\mathbf{R}_{A}\right\rangle_{1 \cup 2 \cup 3},[2]$. The visual comparison of these geometries to $\left\langle\mathbf{R}_{A}\right\rangle_{1}$, clearly showing the shortened bonds, is in Fig. 3 and Fig. 4.

A set of RMSD values for each geometry was computed, comparing it to all the frames used in the averaging procedure to obtain it. The distributions of these sets are shown in Fig. 5, where in the left plot RMSD is calculated using all atoms but methyl hydrogens, while on the right only the $\beta$-ionone ring, excluding its methyl hydrogens, is used.

This shows that basin-based average structures reliably constitute an improvement over direct geometric averaging. This is shown by several different features of their RMSD distribution. Firstly, the peaks of their distributions generally lie at lower RMSD values than those of direct geometric averaging structures and visual inspection of the curves clearly shows that any descriptor such as mean, median, distribution peak would be lower for basin-averaged structures. Secondly, their distribution curves tend to be more narrow than those of direct geometric averaging structures and their distribution curves show a single peak (albeit with shoulders), while those of direct geometric averaging structures show multiple peaks pertaining to the different basins. Even if this features is sometimes not so pronounced if RMSD is calculated for the whole molecule with the only exception of methyl hydrogens, it is always clear when the RMSD calculation is restricted to the moiety involved in the conformerism.

It is worth mentioning that the distribution of RMSD or analogous similarity parameters is a powerful tool to test the validity of averaged structures, because any reasonably important conformational basin which has not been detected will result in a separate peak.

It is also worth noticing that when restricting the RMSD calculation to the sole $\beta$-ionone ring, the distributions for basin-based structures tend to shift to slightly lower values, showing that the $\beta$-ionone ring is not the main contributor to their RMSD values. On the contrary, the peaks of distributions from direct geometric averaging (or some of the peaks when the effect of the lysine tail is significant) shift to higher values, showing that the $\beta$-ionone atoms are actually the largest contributor in the RMSD summation. This feature may, in the future, be used to make the production of basin-based structures automatically, by means of an iterative divisions of the trajectory based on peak recognition in the RMSD distribution.

One may now wonder how basin-averaged structures compare to other geometries from the trajectory. Considering RMSD as a measure of the distance between two structures, a properly averaged structure should have a more favorable RMSD distribution than any of those used to obtain it. Nonetheless, considering that the averaging process is far from trivial, this is an important feature to confirm. To investigate this, 75 random frames where selected from each basin, and their RMSD distributions were compared to that of the basin's averaged structure. As for the previous figures, the RMSD is only calculated between a selected structure and the frames from the conformational basin it belongs to. This is shown in Fig. 6. Analogous figures for the other systems are available in the Supporting Information.

Beside a small cluster around zero, due to the randomly selected geometry itself and a few extremely similar ones, all the RMSD distribution from random frames lie to the right of that of the average structure. This clearly shows that the basin-based geometric averaging procedure is reasonable, and it is better to use basinaveraged structures than a randomly selected structure per basin.

Despite the averaging procedure being performed without direct use of information of the thermodynamics of the system, it is indirectly determined by them, provided the sampling is sufficient: the clusters of values for internal coordinates relate to convex regions of the free energy surface, hence the name "basin". Consequently, our coordinate averaging and the use of minimization of free energy of the supersystem is that, considering that the sampling of the ensemble is performed at a specific temperature and for the full system, the two approaches are related.

Since the division of the trajectory in $K$ con- 

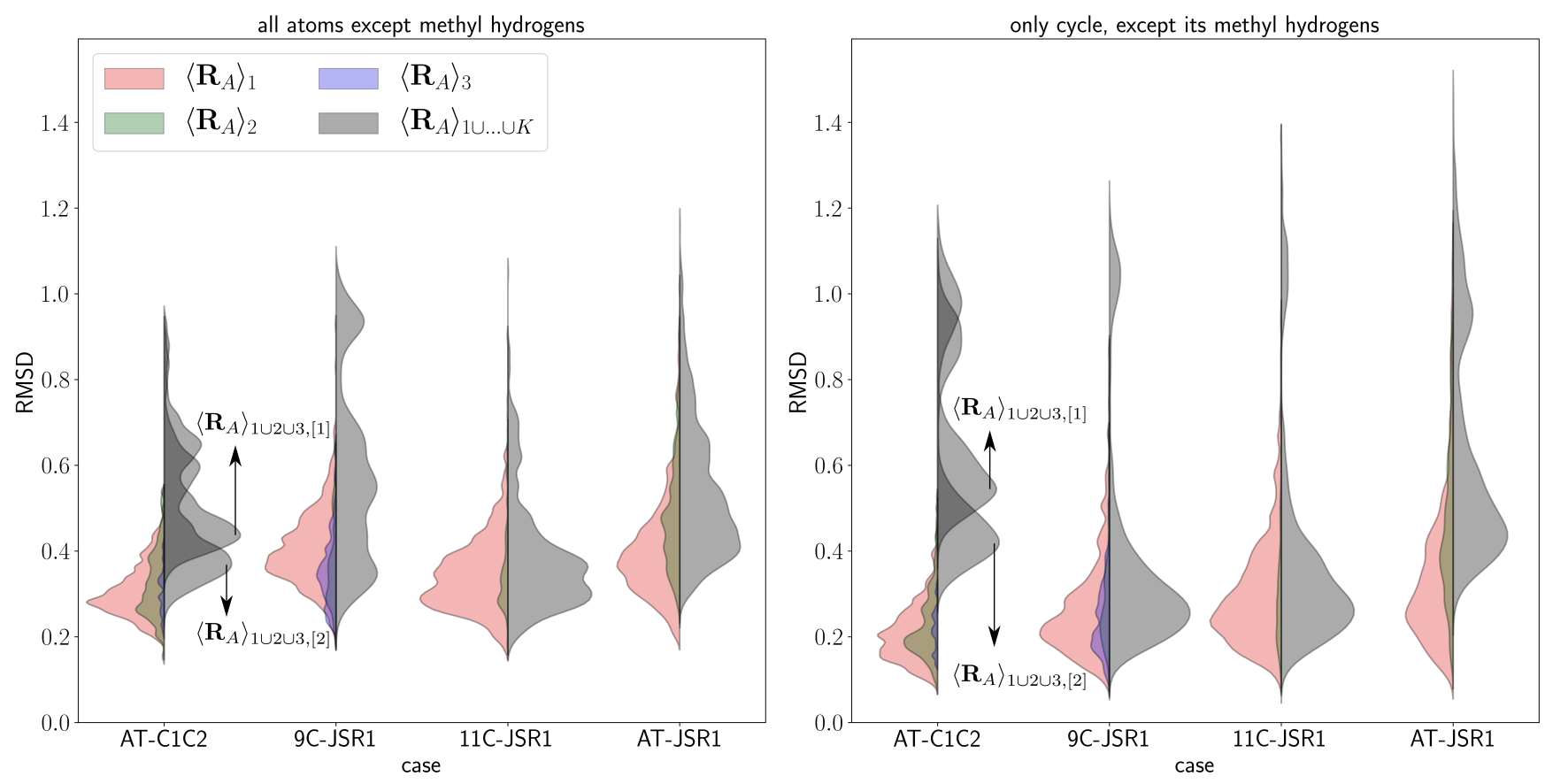

Figure 5: Distribution of RMSD values ( $\Theta$ as defined in Eq. 22) for basin-based(left half of the violins) and direct(right half of the violins) geometric averaging. In the left plot $l$ spans over all atoms but methyl hydrogens, while in the right one it spans only on the $\beta$-ionone ring, with the exclusion of the methyl hydrogens.
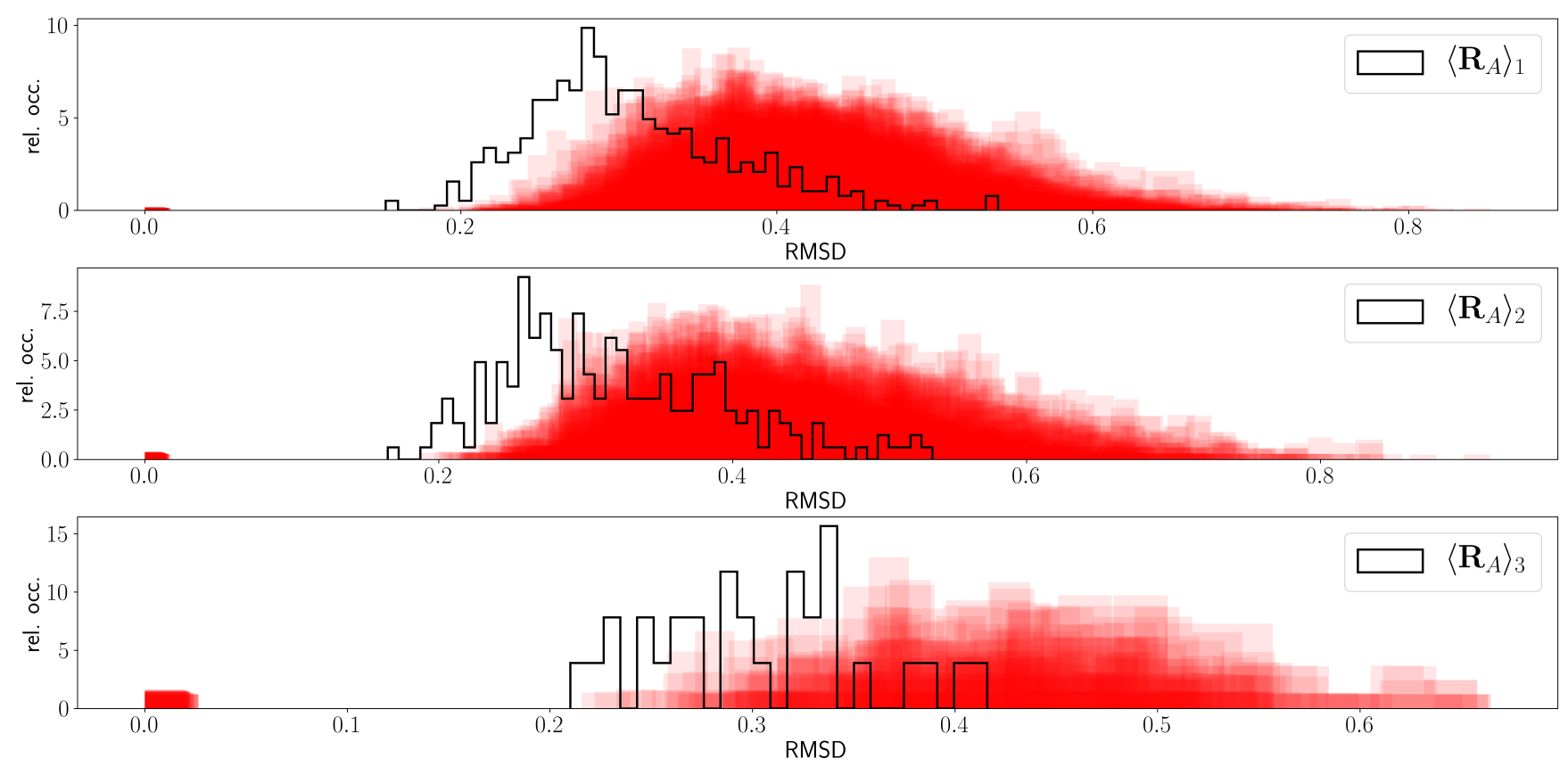

Figure 6: Distribution of RMSD values, calculated for all atoms but methyl hydrogens, for each average structure (black line) and for randomly selected frames (low transparency red bars) for AT-C1C2. 75 random frames were used when possible, while the whole basin (31 frames) was used for 3 . 
formational basins, corresponding to the minimum number of structures to represent the trajectory, is dictated by the thermodynamics of the system, the computational saving is systemdependent. For specific highly flexible systems, the computational saving may potentially be relatively modest. It is nonetheless reasonable to expect a small value of $K$ and hence a large computational advantage for most systems, specifically when embedded in soft condensed matter such as enzymes, rather than in liquids.

\subsection{Observables from ensemble and geometric averaging}

The relationship between a coordinate and a selected observable is far from trivial. Consequently, the sensitivity of an observable on a similarity parameter such as RMSD cannot be foreseen. Nonetheless, there is no guarantee that a poorly representative structure will yield good observables.

After having examined the average solute structures and compared them to those from direct geometric averaging and to randomly selected frames, we can turn to physical observables. We focus our interest on excited states properties, so the first excitation energy and its associated oscillator strength were calculated with ensemble and geometric averaging for every conformational basin, according to Eq. 13 and Eq. 14, and then the weighted averages were obtained according to Eq. 15 and Eq. 16. These excitation energies are shown in Table 1 and Table 2, respectively, while the oscillator strengths are available in the Supporting Information. Table 2 constitutes our principal result. A comparison of the ensemble of observables, ensemble averaged observables, direct and basin-based geometric averaging is available in Fig. 7 (all basins combined) and in the Supporting Information (individual basins).

Methods based on the self-consistent optimization of the solute and solvent such as $\mathrm{ASEP} / \mathrm{MD}^{7,8}$ and ASEC-FEG ${ }^{9,12,14,16,17}$ require geometric convergence of the average structures of the solute and of the solvent for each optimized conformation, requiring
$\sum_{k=1}^{K} N_{\text {cycles }}(k)$ molecular dynamics simulations and $\sum_{k=1}^{K} N_{\text {cycles }}(k)$ quantum mechanical calculations, where $N_{\text {cycles }}(k)$ is the number of cycles necessary for geometric convergence for conformer $k$. Conversely, our approach requires a single sampling of the statistical ensemble via molecular dynamics or molecular Monte Carlo, and $K$ quantum mechanical calculations.

The values obtained from ensemble averaging $\langle\varepsilon\rangle_{N}$ sit in the center of the scatter of the individual values $\varepsilon_{i}$, as would be expected (cf. Fig. 7).

The values from $A T-C 1 C$ 2's $\left\langle\mathbf{R}_{A}\right\rangle_{1 \cup 2 \cup 3,[1]}$ stands out as qualitatively wrong (cf. Table 1). This can be ascribed to the aforementioned artificially shortened bond close to the conjugation chain, causing a larger destabilization in the excited- than in the ground-state, significantly lowering the excitation energy. This is not the case for $\left\langle\mathbf{R}_{A}\right\rangle_{1 \cup 2 \cup 3,[2]}$ (cf. Table 1, Fig. 7).

All direct geometric averaging values $\tilde{\varepsilon}_{1 \cup \ldots \cup K}$, both isolated (iso) and embedded (emb), except that of $\left\langle\mathbf{R}_{A}\right\rangle_{1 \cup 2 \cup 3,[1]}$, are very similar to those from basin-based geometric averaging. This is although specific to our test cases, where the moieties leading to conformerism are not involved in the excitation, and appear not to influence it. Basin-based averaging ought to be preferred nonetheless, as it can be expected to be more robust since it prevents unphysical combinations of dihedrals and angles avoids unphysical undefined bonds such as for $\left\langle\mathbf{R}_{A}\right\rangle_{1 \cup 2 \cup 3,[1]}$. Furthermore, other systems may have conformational basins which strongly influence the chosen observables, either directly via geometric effects, or indirectly if the movement of the environment is coupled to the conformerism, potentially introducing significantly larger errors in the embedded calculation than in the isolated one, affecting the magnitude of the calculated solvatochromic shift.

The isolated solute values (iso) from basinbased geometric averaging within a single basin $\tilde{\varepsilon}_{k}^{i s o}$ seem in good agreement with those from ensemble averaging $\left\langle\varepsilon^{i s o}\right\rangle_{k}$ (cf. Table 1). Basinbased geometric averaging seems to almost consistently (all but one case) overestimate the ex- 
citation energy. At the current state, this cannot be thoroughly explained. Further analyses ought to assess whether this is due to an excessively low ground-state or an excessively high excited state. On the other hand this is not a straightforward task, as ground-state energies are strongly stabilized by having all 5 methyl groups in a favorable position, which in turn should not affect excitation energies significantly. Since the error is very similar in all basins, the weighted averages display the very same trend(cf. Table 2).

The embedded solute values (emb) from geometric averaging $\tilde{\varepsilon}_{k}^{e m b}$ within a single basin also seem in good agreement with those from ensemble averaging, $\left\langle\varepsilon^{e m b}\right\rangle_{k}$. Albeit in some cases almost equal to the reference ensemble averaging value $\left\langle\varepsilon^{e m b}\right\rangle_{k}$, the error varies more than for the isolated solute (iso). There appears to be a slight trend to underestimate the excitation energy. This trend influences the weighted average $\left\langle\tilde{\varepsilon}^{e m b}\right\rangle_{1, \ldots, K}$ to an extent which is due to the weight of the basin with the largest error, as may be expected.

The net effect is that solvatochromic shifts would be slightly underestimated. Specifically, errors on solvatochromic shifts range for individual basins range from $-23.0 \mathrm{meV}$ to -45.5 meV, with a mean absolute error of $34.1 \mathrm{meV}$, and a mean relative error of $21 \%$. Further investigation is necessary to understand to what extent this trend is general to other systems, and what causes it.

Our analysis of FDET-based calculations with averaged liquid environments showed a deterioration of the results below a certain number of frames. ${ }^{21}$ Such a threshold cannot be directly applied to drastically different systems, such as protein environments, where the movement may be different in entity and speed. An excessively low number of frames may be a necessary but not sufficient condition for a relatively large error. If some kind of correlation between the number of frames used to average and the quality of the results were confirmed, one may choose to use a fixed number of frames to average within each basin. In such case the weights, which in this work are obtained from the populations of the subensembles, could also be obtained with some other method such as free energy perturbation theory.

\subsection{Bond length alternation and its effect on excitation energy}

In order to test assessment of geometryobservable relations by means of basin-based geometric averaging, we considered the bond length alternation (BLA) as a collective independent variable. BLA, defined as the difference between the average single bond and the average double bond, has been found to be linearly related to the excitation energies in conjugated polymers. ${ }^{74-76}$ This is the case for our retinal Schiff base, as was observed specifically in $A T-C 1 C 2$ in Ref. 77, as can be seen from the scatter of individual values in Fig. 8 (numerical values available in the Supporting Information). As expected, ensemble averaging within each subensemble yields the same trend as the full scatter. Basin-based geometric averaging was performed in 6 equally spaced BLA-based bins, each divided in 2 based on the $\beta$-ionone ring conformation, while the 31 frames due to the lysine tail's conformerism have been discarded to this end. The excitation energies from basin-based geometric averaging are not exactly equal to those from subensemble averaging, but clearly yield a comparable trend, both for isolated (iso)) and embedded solute $(e m b)$. Namely, there is a clear linear correlation between BLA and excitation energy, with its linear coefficient being slightly larger for the embedded chromophore.

\section{Conclusions}

We introduce an approach for the analysis of internal coordinates to detect conformational basins within which the internal coordinates are averaged. The soundness of the division into basins was assessed by observing the distribution of the root-mean square deviation(RMSD) of the average structure to those that it averages. These distributions are single-peaked.

Observables for the whole ensemble can be modeled as the average of observables for each 
Table 1: Excitation energies from ensemble averaging $\langle\varepsilon\rangle$ vs geometric averaging for single geometries $\tilde{\varepsilon}_{k}$ (cf. Eq. 13). Errors are defined as $\Delta \varepsilon=\tilde{\varepsilon}_{k}-\langle\varepsilon\rangle_{k}$. Excitation energies and errors are given in $\mathrm{eV}$.

\begin{tabular}{c|cccccccc} 
system & $\mathrm{k}$ & $\tilde{\varepsilon}^{i s o}$ & $\left\langle\varepsilon^{i s o}\right\rangle$ & $\tilde{\varepsilon}^{e m b}$ & $\left\langle\varepsilon^{e m b}\right\rangle$ & $\Delta \varepsilon^{i s o}$ & $\Delta \varepsilon^{e m b}$ & $N_{\text {frames }}$ \\
\hline AT-C1C2 & 1 & 3.074 & 3.035 & 3.410 & 3.407 & 0.039 & 0.003 & 497 \\
AT-C1C2 & 2 & 3.082 & 3.059 & 3.415 & 3.437 & 0.023 & -0.022 & 219 \\
AT-C1C2 & 3 & 3.080 & 3.047 & 3.478 & 3.469 & 0.032 & 0.009 & 31 \\
AT-C1C2 & $1 \cup 2 \cup 3,[1]$ & 2.595 & 3.042 & 3.044 & 3.417 & -0.447 & -0.373 & 750 \\
AT-C1C2 & $1 \cup 2 \cup 3,[2]$ & 3.093 & 3.042 & 3.433 & 3.417 & 0.051 & 0.016 & 750 \\
\hline 9C-JSR1 & 1 & 3.074 & 3.049 & 3.174 & 3.176 & 0.025 & -0.002 & 691 \\
9C-JSR1 & 2 & 3.073 & 3.054 & 3.180 & 3.186 & 0.019 & -0.006 & 99 \\
9C-JSR1 & 3 & 3.053 & 3.036 & 3.134 & 3.162 & 0.017 & -0.028 & 165 \\
9C-JSR1 & $1 \cup \cup 3$ & 3.061 & 3.048 & 3.159 & 3.176 & 0.013 & -0.017 & 1000 \\
\hline $11 C-J S R 1$ & 1 & 2.973 & 2.941 & 3.048 & 3.047 & 0.033 & 0.001 & 863 \\
$11 C-J S R 1$ & 2 & 2.882 & 2.898 & 2.964 & 3.011 & -0.016 & -0.047 & 69 \\
$11 C-J S R 1$ & $1 \cup 2$ & 2.971 & 2.938 & 3.047 & 3.044 & 0.033 & 0.002 & 1000 \\
\hline AT-JSR1 & 1 & 2.832 & 2.811 & 2.943 & 2.960 & 0.020 & -0.017 & 741 \\
AT-JSR1 & 2 & 2.904 & 2.870 & 3.016 & 3.023 & 0.034 & -0.007 & 244 \\
AT-JSR1 & $1 \cup 2$ & 2.858 & 2.828 & 2.971 & 2.977 & 0.031 & -0.006 & 1000
\end{tabular}

Table 2: Excitation energies from ensemble averaging $\langle\varepsilon\rangle$ vs geometric averaging for the weighted average of basins $\langle\tilde{f}\rangle_{1, \ldots, K}$ (cf. Eq. 15). Errors are defined as $\Delta \varepsilon=\left\langle\tilde{\varepsilon}_{k}\right\rangle_{1, \ldots, K}-\langle\varepsilon\rangle_{N}$. All values are in $\mathrm{eV}$.

\begin{tabular}{c|ccccccc} 
system & $\mathrm{k}$ & $\left\langle\tilde{\varepsilon}^{i s o}\right\rangle$ & $\left\langle\varepsilon^{i s o}\right\rangle$ & $\left\langle\tilde{\varepsilon}^{e m b}\right\rangle$ & $\left\langle\varepsilon^{e m b}\right\rangle$ & $\Delta \varepsilon^{i s o}$ & $\Delta \varepsilon^{e m b}$ \\
\hline AT-C1C2 & $1,2,3$ & 3.077 & 3.042 & 3.414 & 3.417 & 0.035 & -0.003 \\
\hline 9C-JSR1 & $1,2,3$ & 3.071 & 3.048 & 3.171 & 3.176 & 0.024 & -0.005 \\
\hline 11C-JSR1 & 1,2 & 2.966 & 2.938 & 3.042 & 3.044 & 0.028 & -0.002 \\
\hline AT-JSR1 & 1,2 & 2.851 & 2.828 & 2.962 & 2.977 & 0.023 & -0.015
\end{tabular}



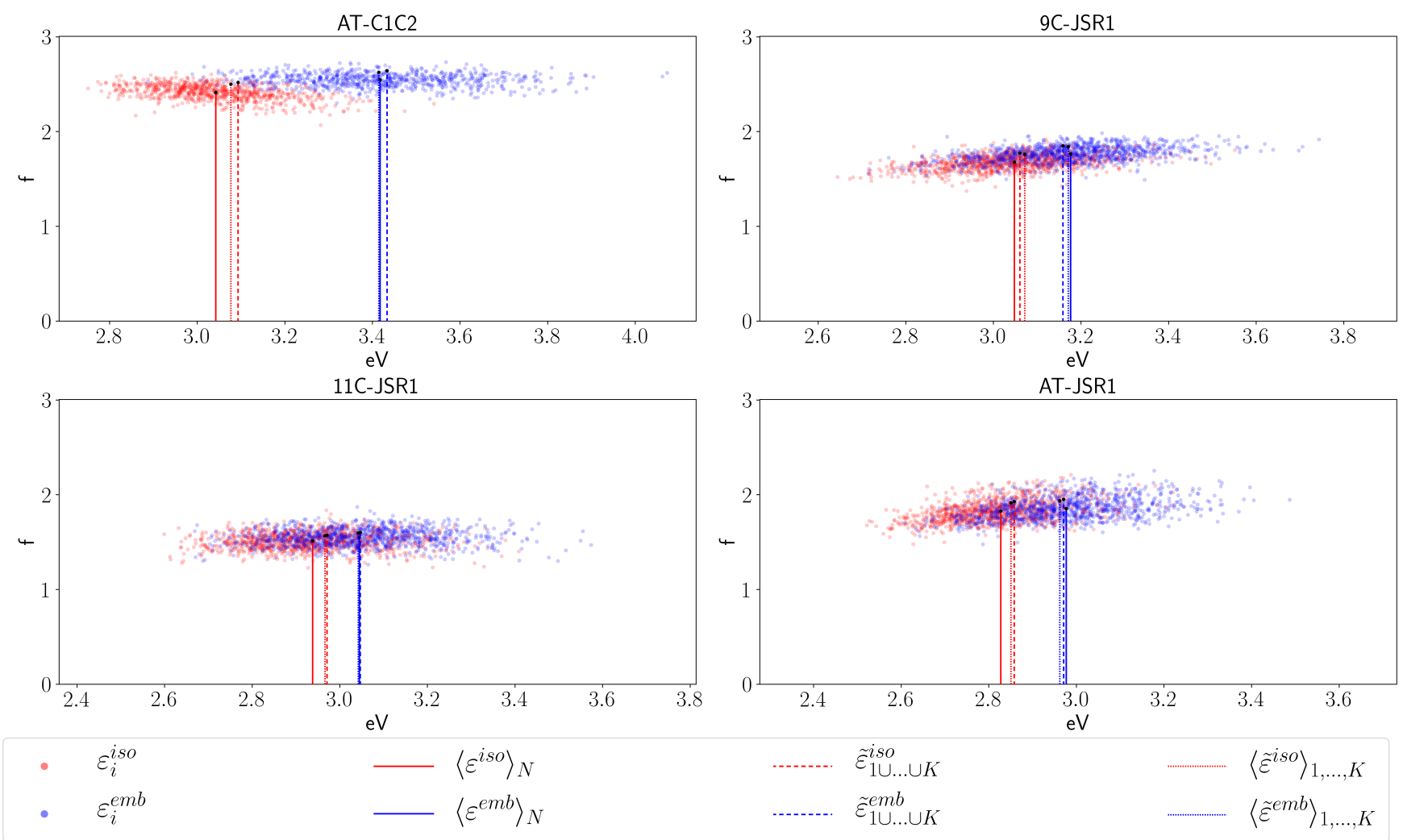

Figure 7: Ensemble, basin-based geometric, and direct geometric averaging for all systems. Legend refers to $\varepsilon$ but applies also to $f$. For $A T$-C1C2, only $\left\langle\mathbf{R}_{A}\right\rangle_{1 \cup 2 \cup 3}[2]$ is shown. The same picture but including the value for $\left\langle\mathbf{R}_{A}\right\rangle_{1 \cup 2 \cup 3}[1]$ is available in the Supporting Information.

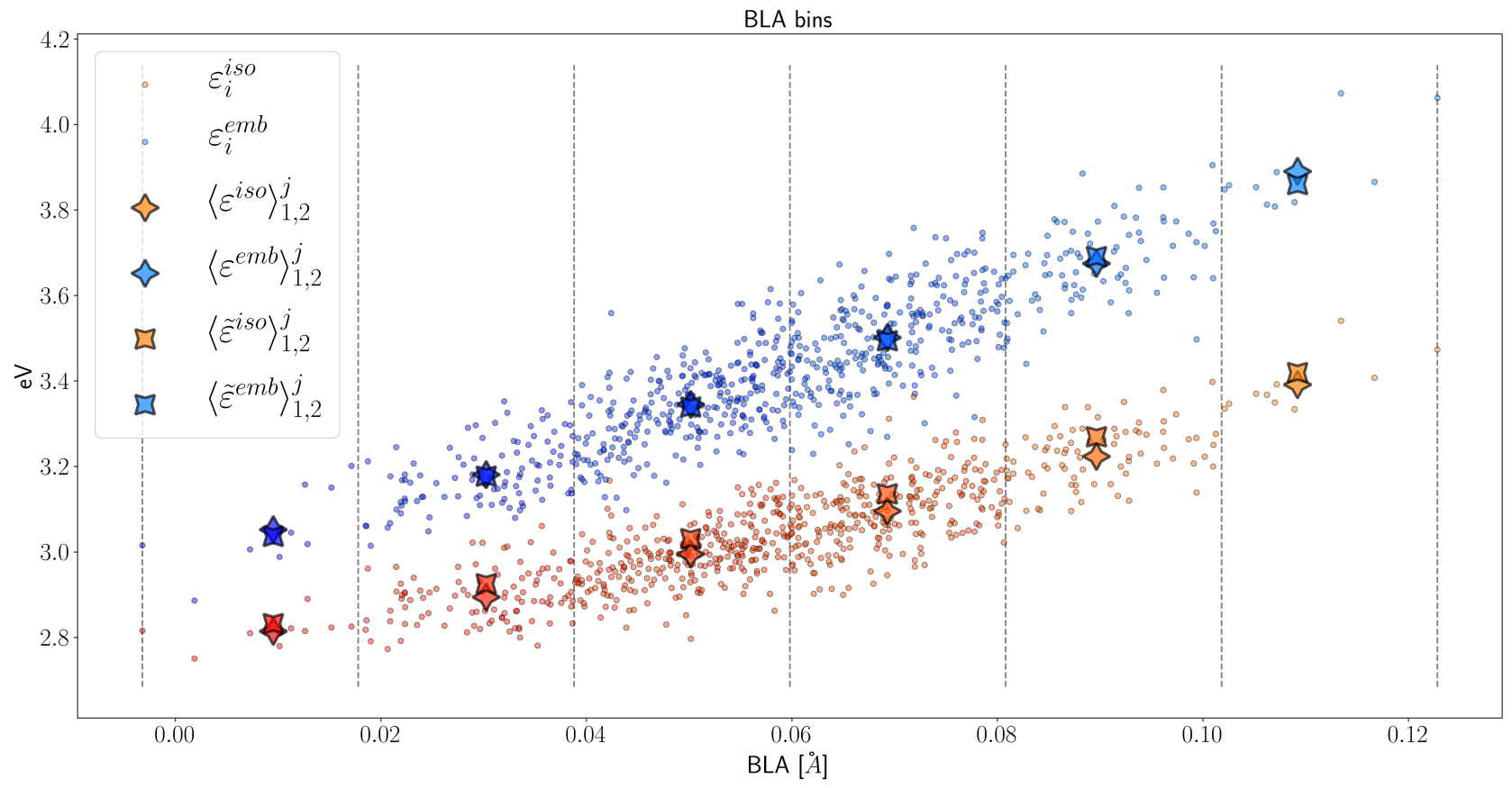

Figure 8: Ensemble vs average geometry, weighted for conformers 1 and 2, within 6 BLA-based bins. Conformer 3 has been neglected in this case. The superscript $j$ in the legend denotes that ensemble and basin-based geometric averaging are restricted to the $j$-th bin. 
average structure, with small errors. This extends environment averaging to systems where multiple conformations are thermally accessible and leads to large computational savings: instead of $N$ quantum calculations, where $N$ is the number of samples of the statistical ensembles, $K$ calculations were performed, where $K$ is the number of detected conformational basins, in our case 2-3.

Another advantage of the proposed procedure is that it does not require self-consistent optimization of the solute and its environment, which for each basin would require multiple sampling procedures and quantum calculations. Only one sampling is required for the full ensemble, and one quantum calculation is performed per basin. The reduction in the required number of calculations may allow the analysis of longer trajectories, larger quantum regions, or the use of higher level electronic structure methods.

Lastly, we applied the same procedure to arbitrary subensembles in order to assess the relation between geometrical parameters and observables.

The concept that this manuscript hinges on, namely that a subensemble of similar solute structures can be represented by its average, may be applied with protocols differing in the clustering techniques, in the observables analyzed, in the type of solute used, and so on and so forth. Albeit thorough analyses of these specific aspects are necessary, we show the importance and the soundness of the main approximation.

Acknowledgement The authors thank the cofunding project of the University of Geneva and the Hebrew University of Jerusalem Israel for financing this collaboration. Igor Schapiro acknowledges the German Research Foundation via the SFB-1078 project C6. Jonathan Richard Church thanks the Zuckerman STEM Leadership Program for their support. Suliman Adam acknowledges the Minerva Stiftung for a post-doctoral fellowship within the framework of the Minerva Fellowship Program.

\section{Supporting Information Avail- able}

The Supporting Information, as mentioned throughout the manuscript, includes:

- visual comparison of the average solute structures for the systems not shown in this manuscript

- distribution of the RMSD values for randomly selected frames for the systems not shown in this manuscript

- a table with the oscillator strengths values for ensemble and geometric averaging

- spectra for individual basins for each system

- numerical values related to Fig. 8

\section{References}

(1) Tomasi, J.; Persico, M. Molecular Interactions in Solution: An Overview of Methods Based on Continuous Distributions of the Solvent. Chem. Rev. 1994, 94, 20272094.

(2) Tomasi, J.; Mennucci, B.; Cammi, R. Quantum Mechanical Continuum Solvation Models. Chem. Rev. 2005, 105, 2999 3094 .

(3) Klamt, A.; Schüürmann, G. COSMO: a new approach to dielectric screening in solvents with explicit expressions for the screening energy and its gradient. $J$. Chem. Soc., Perkin Trans. 2 1993, 799805.

(4) Galván, I.; Sánchez, M.; Martín, M.; Olivares del Valle, F.; Aguilar, M. ASEP/MD: A program for the calculation of solvent effects combining QM/MM methods and the mean field approximation. Comput. Phys. Commun. 2003, 155, 244-259. 
(5) Sanchez, M. L.; A., A. M.; Olivares del Valle, F. J. Study of solvent effects by means of averaged solvent electrostatic potentials obtained from molecular dynamics data. J. Comput. Chem. 1997, 18, 313322 .

(6) Sanchez Mendoza, M. L.; Aguilar, M. A.; Olivares Del Valle, F. J. A mean field approach that combines quantum mechanics and molecular dynamics simulation: The water molecule in liquid water. Journal of Molecular Structure: THEOCHEM 1998, 426, 181-190.

(7) Martín, M. E.; Sánchez, M. L.; Olivares del Valle, F. J.; Aguilar, M. A. A multiconfiguration self-consistent field/molecular dynamics study of the $\left(\mathrm{n} \pi^{*}\right) 1$ transition of carbonyl compounds in liquid water. The Journal of Chemical Physics 2000, 113, 6308-6315.

(8) Sánchez, M. L.; Martín, M. E.; Aguilar, M. A.; Olivares Del Valle, F. J. Solvent Effects by Means of Averaged Solvent Electrostatic Potentials: Coupled Method. Journal of Computational Chemistry 2000, 21, 705-715.

(9) Dratch, B. D.; Orozco-Gonzalez, Y.; Gadda, G.; Gozem, S. Ionic Atmosphere Effect on the Absorption Spectrum of a Flavoprotein: A Reminder to Consider Solution Ions. Journal of Physical Chemistry Letters 2021, 12, 8384-8396.

(10) Nikolaev, D. M.; Manathunga, M.; Orozco-Gonzalez, Y.; Shtyrov, A. A.; Guerrero Martínez, Y. O.; Gozem, S.; Ryazantsev, M. N.; Coutinho, K.; Canuto, S.; Olivucci, M. Free Energy Computation for an Isomerizing Chromophore in a Molecular Cavity via the Average Solvent Electrostatic Configuration Model: Applications in Rhodopsin and Rhodopsin-Mimicking Systems. Journal of Chemical Theory and Computation 2021, 17, 5885-5895.
(11) Coutinho, K.; Georg, H.; Fonseca, T.; Ludwig, V.; Canuto, S. An efficient statistically converged average configuration for solvent effects. Chem. Phys. Lett. 2007, 437, 148-152.

(12) Orozco-Gonzalez, Y.; Manathunga, M.; Marín, M. d. C.; Agathangelou, D.; Jung, K.-H.; Melaccio, F.; Ferré, N.; Haacke, S.; Coutinho, K.; Canuto, S.; Olivucci, M. An Average Solvent Electrostatic Configuration Protocol for QM/MM Free Energy Optimization: Implementation and Application to Rhodopsin Systems. J. Chem. Theory Comput. 2017, 13, 6391-6404.

(13) de Almeida, A. R.; Oliveira, L. B. A.; Colherinhas, G. Solvent effects on the spectroscopic properties of Damascone derivatives: A sequential Monte Carlo/Quantum Mechanics study. Chemical Physics Letters 2019, 730, 531-537.

(14) Bistafa, C.; Georg, H. C.; Canuto, S. Combining ab initio multiconfigurational and Free Energy Gradient methods to study the $\pi \rightarrow \pi^{*}$ excited state structure and properties of uracil in water. Computational and Theoretical Chemistry 2014, 1040-1041, 312-320, Excited states: From isolated molecules to complex environments.

(15) Jaramillo, P.; Coutinho, K.; Cabral, B. J.; Canuto, S. Explicit solvent effects on the visible absorption spectrum of a photosynthetic pigment: Chlorophyll-c2 in methanol. Chemical Physics Letters 2011, 516, 250-253.

(16) Torres, E.; Georg, H.; Fonseca, T.; Castro, M. First hyperpolarizability of isomers of pyridinium N-phenoxide betaine dye in solution using the ASEC-FEG method. Chemical Physics Letters 2018, 699, 261-266.

(17) Brandão, I.; Fonseca, T. L.; Georg, H. C.; Castro, M. A.; Pontes, R. B. Assessing 
the structure and first hyperpolarizability of Li@B10H14 in solution: a sequential QM/MM study using the ASECFEG method. Phys. Chem. Chem. Phys. 2020, 22, 17314-17324.

(18) Wesołowski, T. A. Embedding a multideterminantal wave function in an orbitalfree environment. Phys. Rev. A 2008, 77, 012504 .

(19) Wesolowski, T. A.; Warshel, A. Frozen density functional approach for ab initio calculations of solvated molecules. $J$. Phys. Chem. 1993, 97, 8050-8053.

(20) Kaminski, J. W.; Gusarov, S.; Wesolowski, T. A.; Kovalenko, A. Modeling Solvatochromic Shifts Using the Orbital-Free Embedding Potential at Statistically Mechanically Averaged Solvent Density. J. Phys. Chem. A 2010, 114, 6082-6096.

(21) González-Espinoza, C. E.; Rumble, C. A.; Borgis, D.; Wesolowski, T. A. Quantifying Fluctuations of Average Environments for Embedding Calculations. In preparation 2021, XX-XX.

(22) Shedge, S. V.; Wesolowski, T. A. Nonuniform Continuum Model for Solvatochromism Based on Frozen-Density Embedding Theory. ChemPhysChem 2014, 15, 3291-3300.

(23) Shedge, S. V.; Zhou, X.; Wesolowski, T. A. Non-uniform continuum model for solvated species based on Frozen-Density Embedding Theory: The study case of solvatochromism of coumarin 153. Chimia 2014, 68, 609-614.

(24) Laktionov, A. A.; Chemineau-Chalaye, E.; Wesolowski, T. A. Frozen-density embedding theory with average solvent charge densities from explicit atomistic simulations. Journal of Chemical Theory and Computation 2016, 18, 2829-2844.

(25) Beveridge, D. L.; Schnuelle, G. W. Statistical thermodynamic consideration of solvent effects on conformational stability. The supermolecule-continuum model. Journal of Physical Chemistry 1974, 78, 2064-2069.

(26) Hodes, Z. I.; Némethy, G.; Scheraga, H. A. Model for the conformational analysis of hydrated peptides. Effect of hydration on the conformational stability of the terminally blocked residues of the 20 naturally occurring amino acids. Biopolymers 1979, $18,1565-1610$.

(27) Cappelli, C.; Corni, S.; Tomasi, J. Solvent effects on trans/gauche conformational equilibria of substituted chloroethanes: A polarizable continuum model study. Journal of Physical Chemistry A 2001, 105, 10807-10815.

(28) González-Alemán, R.; HernándezCastillo, D.; Caballero, J.; MonteroCabrera, L. A. Quality Threshold Clustering of Molecular Dynamics: A Word of Caution. Journal of Chemical Information and Modeling 2020, 60, 467-472.

(29) Melvin, R. L.; Godwin, R. C.; Xiao, J.; Thompson, W. G.; Berenhaut, K. S.; Salsbury, F. R. Uncovering Large-Scale Conformational Change in Molecular Dynamics without Prior Knowledge. Journal of Chemical Theory and Computation 2016, 12, 6130-6146.

(30) De Paris, R.; Quevedo, C. V.; Ruiz, D. D.; Norberto De Souza, O.; Barros, R. C. Clustering molecular dynamics trajectories for optimizing docking experiments. Computational Intelligence and Neuroscience 2015, 2015.

(31) Shao, J.; Tanner, S. W.; Thompson, N.; Cheatham, T. E. I. Clustering molecular dynamics trajectories: 1. Characterizing the performance of different clustering algorithms. Journal of Chemical Theory and Computation 2007, 3, 2312-2334. 
(32) Sullivan, D. C.; Lim, C. Quantifying polypeptide conformational space: Sensitivity to conformation and ensemble definition. Journal of Physical Chemistry B 2006, 110, 16707-16717.

(33) Feher, M.; Schmidt, J. M. Fuzzy clustering as a means of selecting representative conformers and molecular alignments. Journal of Chemical Information and Computer Sciences 2003, 43, 810-818.

(34) Clark, C. A.; Mezei, M.; Murphy, R. F.; Lovas, S. Conformational space comparison of GnRH and lGnRH-III using molecular dynamics, cluster analysis and Monte Carlo thermodynamic integration. Journal of Biomolecular Structure and Dynamics 2001, 18, 733-748.

(35) Gabarro-Arpa, J.; Revilla, R. Clustering of a molecular dynamics trajectory with a Hamming distance. Computers and Chemistry 2000, 24, 693-698.

(36) Daura, X.; Van Gunsteren, W. F.; Mark, A. E. Folding-unfolding thermodynamics of a $\beta$-heptapeptide from equilibrium simulations. Proteins: Structure, Function and Genetics 1999, 34, 269-280.

(37) Heyer, L. J.; Kruglyak, S.; Yooseph, S. Exploring expression data identification and analysis of coexpressed genes. Genome Research 1999, 9, 1106-1115.

(38) Troyer, J. M.; Cohen, F. E. Protein conformational landscapes: Energy minimization and clustering of a long molecular dynamics trajectory. Proteins: Structure, Function, and Bioinformatics 1995, 23, 97-110.

(39) Shenkin, P. S.; Mcdonald, D. Q. Cluster analysis of molecular conformations. Journal of Computational Chemistry 1994, 15, 899-916.

(40) Torda, A. E.; Gunsteren, W. F. V. Algorithms for Clustering Molecular Dynamics Configurations. Journal of Computational Chemistry 1994, 15, 1331-1340.
(41) Karpen, M. E.; Tobias, D. J.; Brooks, C. L. Statistical Clustering Techniques for the Analysis of Long Molecular Dynamics Trajectories: Analysis of 2.2-ns Trajectories of YPGDV. Biochemistry 1993, 32, 412-420.

(42) Michel, A. G.; Jeandenans, C. Multiconformational investigations of polypeptidic structures, using clustering methods and principal components analysis. Computers and Chemistry 1993, 17, 49-59.

(43) Perkins, T. D.; Dean, P. M. An exploration of a novel strategy for superposing several flexible molecules. Journal of Computer-Aided Molecular Design 1993, ๆ, 155-172.

(44) Gordon, H. L.; Somorjai, R. L. Fuzzy cluster analysis of molecular dynamics trajectories. Proteins: Structure, Function, and Bioinformatics 1992, 14, 249-264.

(45) Murray-Rust, P.; Raftery, J. Computer analysis of molecular geometry. Part VI: Classification of differences in conformation. Journal of Molecular Graphics $\mathbf{1 9 8 5}$, 3, 50-59.

(46) Marchionini, C.; Maigret, B.; Prémilat, S. Models for the conformational behaviour of Angiotensin-II in acidic aqueous solutions. Biochemical and Biophysical Research Communications 1983, 112, 339346 .

(47) Kato, H. E.; Zhang, F.; Yizhar, O.; Ramakrishnan, C.; Nishizawa, T.; Hirata, K.; Ito, J.; Aita, Y.; Tsukazaki, T.; Hayashi, S.; Hegemann, P.; Maturana, A. D.; Ishitani, R.; Deisseroth, K.; Nureki, O. Crystal structure of the channelrhodopsin light-gated cation channel. Nature 2012, 482, 369-374.

(48) Adam, S.; Bondar, A. N. Mechanism by which water and protein electrostatic interactions control proton transfer at the active site of channelrhodopsin. PLoS ONE 2018, 13, 1-28. 
(49) Church, J. R.; Olsen, J. M. H.; Schapiro, I. The Impact of Retinal Configuration on Protein-Chromophore Interactions in Bistable Jumping Spider Rhodopsin-1. In preparation $\mathrm{XX}-\mathrm{XX}$.

(50) Varma, N.; Mutt, E.; Mühle, J.; Panneels, V.; Terakita, A.; Deupi, X.; Nogly, P.; Schertler, G. F.; Lesca, E. Crystal structure of jumping spider rhodopsin1 as a light sensitive GPCR. Proceedings of the National Academy of Sciences of the United States of America 2019, 116, 14547-14556.

(51) García-Prieto, F. F.; Galván, I. F.; Aguilar, M. A.; Martn, M. E. Study on the conformational equilibrium of the alanine dipeptide in water solution by using the averaged solvent electrostatic potential from molecular dynamics methodology. Journal of Chemical Physics 2011, 135.

(52) Mobian, P.; Nicolas, C.; Francotte, E.; Bürgi, T.; Lacour, J. Synthesis, resolution, and VCD analysis of an enantiopure diazaoxatricornan derivative. Journal of the American Chemical Society 2008, 130, 6507-6514.

(53) Kabsch, W. A discussion of the solution for the best rotation to relate two sets of vectors. Acta Crystallographica Section A 1978, 34, 827-828.

(54) Kabsch, W. A solution for the best rotation to relate two sets of vectors. Acta Crystallographica Section A 1976, 32, 922-923.

(55) Zech, A.; Aquilante, F.; Wesolowski, T. A. Orthogonality of embedded wave functions for different states in frozen-density embedding theory. J. Chem. Phys. 2015, $143,164106$.

(56) Wesolowski, T. A. Embedding potentials for excited states of embedded species. $J$. Chem. Phys. 2014, 140, 18A530.
(57) Epifanovsky, E.; Gilbert, A. T. B.; Feng, X.; Lee, J.; Mao, Y.; Mardirossian, N.; Pokhilko, P.; White, A. F.; Coons, M. P.; Dempwolff, A. L.; Gan, Z.; Hait, D.; Horn, P. R.; Jacobson, L. D.; Kaliman, I.; Kussmann, J.; Lange, A. W.; Lao, K. U.; Levine, D. S.; Liu, J.; McKenzie, S. C.; Morrison, A. F.; Nanda, K. D.; Plasser, F.; Rehn, D. R.; Vidal, M. L.; You, Z.-Q.; Zhu, Y.; Alam, B.; Albrecht, B. J.; Aldossary, A.; Alguire, E.; Andersen, J. H.; Athavale, V.; Barton, D.; Begam, K.; Behn, A.; Bellonzi, N.; Bernard, Y. A.; Berquist, E. J.; Burton, H. G. A.; Carreras, A.; CarterFenk, K.; Chakraborty, R.; Chien, A. D.; Closser, K. D.; Cofer-Shabica, V.; Dasgupta, S.; de Wergifosse, M.; Deng, J.; Diedenhofen, M.; Do, H.; Ehlert, S.; Fang, P.-T.; Fatehi, S.; Feng, Q.; Friedhoff, T.; Gayvert, J.; Ge, Q.; Gidofalvi, G.; Goldey, M.; Gomes, J.; González-Espinoza, C. E.; Gulania, S.; Gunina, A. O.; Hanson-Heine, M. W. D.; Harbach, P. H. P.; Hauser, A.; Herbst, M. F.; Hernndez Vera, M.; Hodecker, M.; Holden, Z. C.; Houck, S.; Huang, X.; Hui, K.; Huynh, B. C.; Ivanov, M.; Jász, A.; Ji, H.; Jiang, H.; Kaduk, B.; Kähler, S.; Khistyaev, K.; Kim, J.; Kis, G.; Klunzinger, P.; KoczorBenda, Z.; Koh, J. H.; Kosenkov, D.; Koulias, L.; Kowalczyk, T.; Krauter, C. M.; Kue, K.; Kunitsa, A.; Kus, T.; Ladjnszki, I.; Landau, A.; Lawler, K. V.; Lefrancois, D.; Lehtola, S.; Li, R. R.; Li, Y.-P.; Liang, J.; Liebenthal, M.; Lin, H.-H.; Lin, Y.-S.; Liu, F.; Liu, K.Y.; Loipersberger, M.; Luenser, A.; Manjanath, A.; Manohar, P.; Mansoor, E.; Manzer, S. F.; Mao, S.-P.; Marenich, A. V.; Markovich, T.; Mason, S.; Maurer, S. A.; McLaughlin, P. F.; Menger, M. F. S. J.; Mewes, J.-M.; Mewes, S. A.; Morgante, P.; Mullinax, J. W.; Oosterbaan, K. J.; Paran, G.; Paul, A. C.; Paul, S. K.; Pavoevi, F.; Pei, Z.; Prager, S.; Proynov, E. I.; 
Rák, A.; Ramos-Cordoba, E.; Rana, B.; Rask, A. E.; Rettig, A.; Richard, R. M.; Rob, F.; Rossomme, E.; Scheele, T.; Scheurer, M.; Schneider, M.; Sergueev, N.; Sharada, S. M.; Skomorowski, W.; Small, D. W.; Stein, C. J.; Su, Y.-C.; Sundstrom, E. J.; Tao, Z.; Thirman, J.; Tornai, G. J.; Tsuchimochi, T.; Tubman, N. M.; Veccham, S. P.; Vydrov, O.; Wenzel, J.; Witte, J.; Yamada, A.; Yao, K.; Yeganeh, S.; Yost, S. R.; Zech, A.; Zhang, I. Y.; Zhang, X.; Zhang, Y.; Zuev, D.; Aspuru-Guzik, A.; Bell, A. T.; Besley, N. A.; Bravaya, K. B.; Brooks, B. R.; Casanova, D.; Chai, J.-D.; Coriani, S.; Cramer, C. J.; Cserey, G.; DePrince, A. E.; DiStasio, R. A.; Dreuw, A.; Dunietz, B. D.; Furlani, T. R.; Goddard, W. A.; Hammes-Schiffer, S.; Head-Gordon, T.; Hehre, W. J.; Hsu, C.P.; Jagau, T.-C.; Jung, Y.; Klamt, A.; Kong, J.; Lambrecht, D. S.; Liang, W.; Mayhall, N. J.; McCurdy, C. W.; Neaton, J. B.; Ochsenfeld, C.; Parkhill, J. A.; Peverati, R.; Rassolov, V. A.; Shao, Y.; Slipchenko, L. V.; Stauch, T.; Steele, R. P.; Subotnik, J. E.; Thom, A. J. W.; Tkatchenko, A.; Truhlar, D. G.; Van Voorhis, T.; Wesolowski, T. A.; Whaley, K. B.; Woodcock, H. L.; Zimmerman, P. M.; Faraji, S.; Gill, P. M. W.; Head-Gordon, M.; Herbert, J. M.; Krylov, A. I. Software for the frontiers of quantum chemistry: An overview of developments in the Q-Chem 5 package. The Journal of Chemical Physics 2021, 155, 084801.

(58) Prager, S.; Zech, A.; Aquilante, F.; Dreuw, A.; Wesolowski, T. A. First time combination of frozen density embedding theory with the algebraic diagrammatic construction scheme for the polarization propagator of second order. J. Chem. Phys. 2016, 144, 204103.

(59) Thomas, L. H. The calculation of atomic fields. Math. Proc. Cambridge Philos. Soc. 1927, 23, 542.
(60) Fermi, E. Eine statistische Methode zur Bestimmung einiger Eigenschaften des Atoms und ihre Anwendung auf die Theorie des periodischen Systems der Elemente. Z. Phys. 1928, 48, 73-79.

(61) Slater, J. C. The Theory of Complex Spectra. Phys. Rev. 1929, 34, 1293-1322.

(62) Vosko, S. H.; Wilk, L.; Nusair, M. Accurate spin-dependent electron liquid correlation energies for local spin density calculations: a critical analysis. Can. J. Phys. 1980, 58, 1200-1211.

(63) MacKerell, A. D.; Bashford, D.; Bellott, M.; Dunbrack, R. L.; Evanseck, J. D.; Field, M. J.; Fischer, S.; Gao, J.; Guo, H.; Ha, S.; Joseph-McCarthy, D.; Kuchnir, L.; Kuczera, K.; Lau, F. T.; Mattos, C.; Michnick, S.; Ngo, T.; Nguyen, D. T.; Prodhom, B.; Reiher, W. E.; Roux, B.; Schlenkrich, M.; Smith, J. C.; Stote, R.; Straub, J.; Watanabe, M.; WiórkiewiczKuczera, J.; Yin, D.; Karplus, M. Allatom empirical potential for molecular modeling and dynamics studies of proteins. Journal of Physical Chemistry B 1998, 102, 3586-3616.

(64) Mackerell, A. D.; Feig, M.; Brooks, C. L. Extending the treatment of backbone energetics in protein force fields: Limitations of gas-phase quantum mechanics in reproducing protein conformational distributions in molecular dynamics simulation. Journal of Computational Chemistry 2004, 25, 1400-1415.

(65) Maier, J. A.; Martinez, C.; Kasavajhala, K.; Wickstrom, L.; Hauser, K. E.; Simmerling, C. ff14SB: Improving the Accuracy of Protein Side Chain and Backbone Parameters from ff99SB. Journal of Chemical Theory and Computation 2015, 11, 3696-3713.

(66) Schirmer, J. Beyond the random-phase approximation: A new approximation scheme for the polarization propagator. Phys. Rev. A 1982, 26, 2395-2416. 
(67) Dunning, T. H. Gaussian basis sets for use in correlated molecular calculations. I. The atoms boron through neon and hydrogen. J. Chem. Phys. 1989, 90, 1007-1023.

(68) Weigend, F.; Köhn, A.; Hättig, C. Efficient use of the correlation consistent basis sets in resolution of the identity MP2 calculations. Journal of Chemical Physics 2002, 116, 3175-3183.

(69) Weigend, F.; Häser, M.; Patzelt, H.; Ahlrichs, R. RI-MP2: Optimized auxiliary basis sets and demonstration of efficiency. Chemical Physics Letters 1998, 294, 143152.

(70) Weser, O. An efficient and general library for the definition and use of internal coordinates in large molecular systems. M.Sc. thesis, Georg August Universität Göttingen, 2017.

(71) Pelleg, D.; Moore, A. X-means: Extending K-means with Efficient Estimation of the Number of Clusters. In Proceedings of the 17th International Conf. on Machine Learning. 2000; pp 727-734.

(72) Novikov, A. V. PyClustering: Data Mining Library. Journal of Open Source Software 2019, 4, 1230 .

(73) González-Espinoza, C. E.; Ricardi, N. FDET-Averaged. https://github.com/ crisely09/fdeta, 2020.

(74) Yang, S.; Kertesz, M. Bond length alternation and energy band gap of polyyne. Journal of Physical Chemistry A 2006, 110, 9771-9774.

(75) Hoffmann, M.; Wanko, M.; Strodel, P.; König, P. H.; Frauenheim, T.; Schulten, K.; Thiel, W.; Tajkhorshid, E.; Elstner, M. Color tuning in rhodopsins: The mechanism for the spectral shift between bacteriorhodopsin and sensory rhodopsin II. Journal of the American Chemical Society 2006, 128, 10808-10818.
(76) Choi, C. H.; Kertesz, M.; Karpfen, A. The effects of electron correlation on the degree of bond alternation and electronic structure of oligomers of polyacetylene. Journal of Chemical Physics 1997, 10\%, 67126721.

(77) Adam, S.; Wiebeler, C.; Schapiro, I. Structural Factors Determining the Absorption Spectrum of Channelrhodopsins: A Case Study of the Chimera C1C2. Journal of Chemical Theory and Computation 2021, 2. 
Graphical TOC Entry

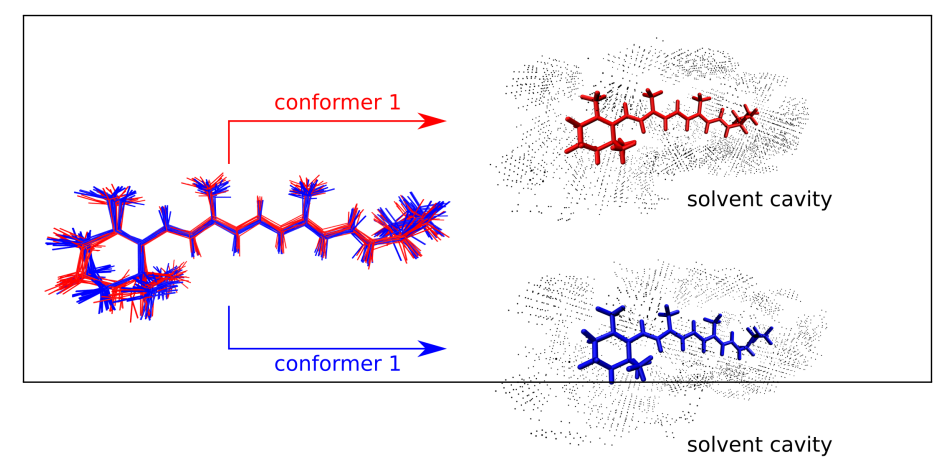

